Interesting Images

\title{
Rapid Evolution of an Aortic Endocarditis
}

\author{
Gaetano Todde, Paola Gargiulo, Grazia Canciello, Felice Borrelli, Emanuele Pilato, Giovanni Esposito \\ and Maria Angela Losi *
}

check for updates

Citation: Todde, G.; Gargiulo, P.; Canciello, G.; Borrelli, F.; Pilato, E.; Esposito, G.; Losi, M.A. Rapid Evolution of an Aortic Endocarditis. Diagnostics 2022, 12, 327. https:/ /doi.org/10.3390/ diagnostics 12020327

Academic Editor: Fabien Hyafil

Received: 23 December 2021

Accepted: 26 January 2022

Published: 27 January 2022

Publisher's Note: MDPI stays neutral with regard to jurisdictional claims in published maps and institutional affiliations.

Copyright: (C) 2022 by the authors. Licensee MDPI, Basel, Switzerland. This article is an open access article distributed under the terms and conditions of the Creative Commons Attribution (CC BY) license (https:// creativecommons.org/licenses/by/ $4.0 /)$.
Department of Advanced Biomedical Sciences, University Federico II, 80131 Naples, Italy; gaetano.todde@virgilio.it (G.T.); paola.gargiulo@unina.it (P.G.); grazia.canciello@hotmail.it (G.C.); feliceborrelli@yahoo.it (F.B.); emapilato@yahoo.it (E.P.); espogiov@unina.it (G.E.)

* Correspondence: losi@unina.it; Tel.: +39-081-746-22-21

Abstract: Cardiac surgery is necessary in almost $50 \%$ of patients with endocarditis. Early surgery, i.e., the surgery performed during the first hospitalization, is required in the following cases: heart failure secondary to valve regurgitation; $S$. aureus, fungal organism, or other highly resistant organism infection; heart block, annular or aortic abscess, or destructive penetrating lesions; evidence of persistent infection as manifested by persistent bacteremia or fevers lasting $>5$ days after onset of appropriate antimicrobial therapy. A 62-year-old man developed a fever $\left(38^{\circ} \mathrm{C}\right) 3$ days after a transaortic electrophysiological study; blood cultures were positive for $S$. aureus, and were sensitive to vancomycin and ceftaroline. Antibiotic therapy was started, controlling the fever and the patient's infective and inflammatory profiles well; however, 3 days later, acute aortic regurgitation developed. At transesophageal echocardiography (TEE), a rare condition was revealed-vegetation was attached to the aortic wall, impeding correct aortic valve closure. Cardiac operation was carried out and the time for surgery was discussed; based on the patient's clinically stable condition, and on the infection, which was controlled well by antibiotics therapy, surgery was not performed in emergency circumstance (within 24-48 h)—rather, it was programmed during the hospitalization. A TEE surveillance was initiated, and after 7 days, TEE revealed a new picture, with images of an aortic abscess with small perforation in the right atrium, requiring emergency surgery, carried out $20 \mathrm{~h}$ later. In our case, the rapid evolution of the vegetation attached to the aortic wall suggested the following: (1) that the time for the surgery cannot be guided only by clinical procedure but must also be guided by imaging pictures; (2) that strictly TEE surveillance is mandatory in patients with aortic endocarditis not initially referred for emergency surgery.

Keywords: endocarditis; acute aortic regurgitation; transesophageal echocardiography; cardiac surgery
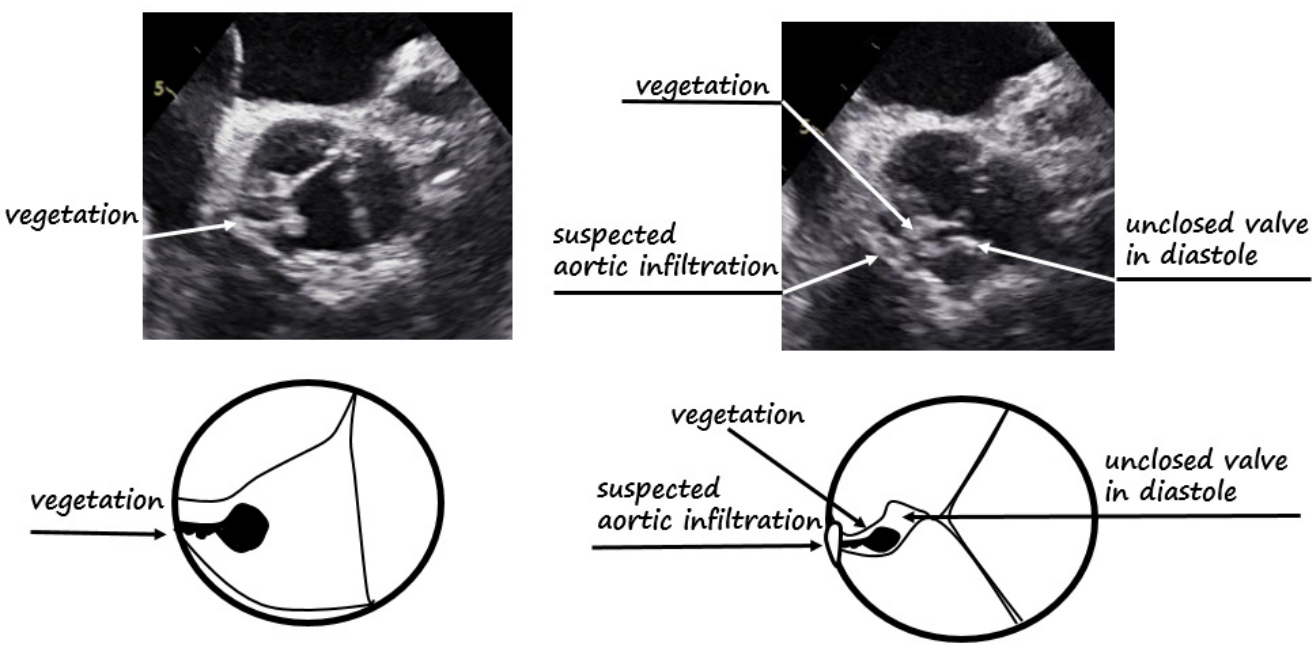

Figure 1. First TEE at the time of acute aortic regurgitation presentation. Top left panel shows TEE at level of aortic valve; a shaggy, pedunculated mass [1] is attached to the aortic wall; bottom left panel 
shows the relative scheme. Top right panel shows TEE at same level in diastole, with vegetation impeding correct valve closure and a suspected aortic wall infiltration; bottom right panel shows the relative pictogram.
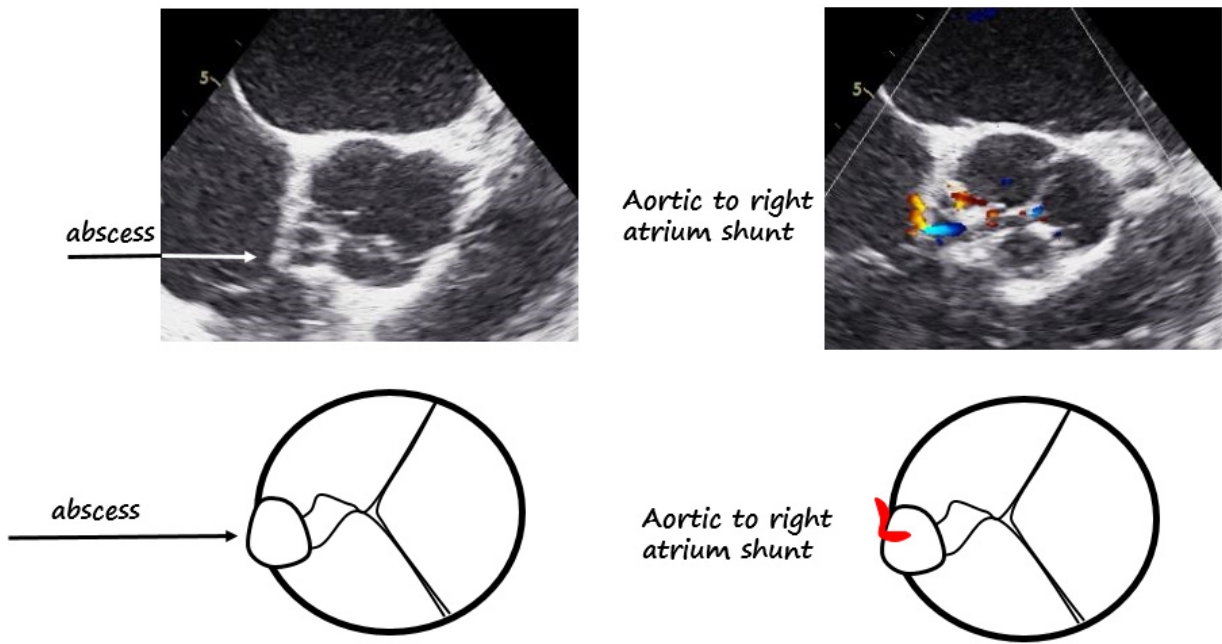

Figure 2. Second TEE, 7 days later. Top left panel shows aortic wall abscess [2]; bottom left panel shows the relative scheme. Top right panel shows TEE at same level with color Doppler, demonstrating a little aortic to the right of the atrium shunt; bottom right panel shows the relative pictogram.

aortic abscess forced in the aortic wall by a Klemmer forceps, introduced through right atrium; a little fissuration in present

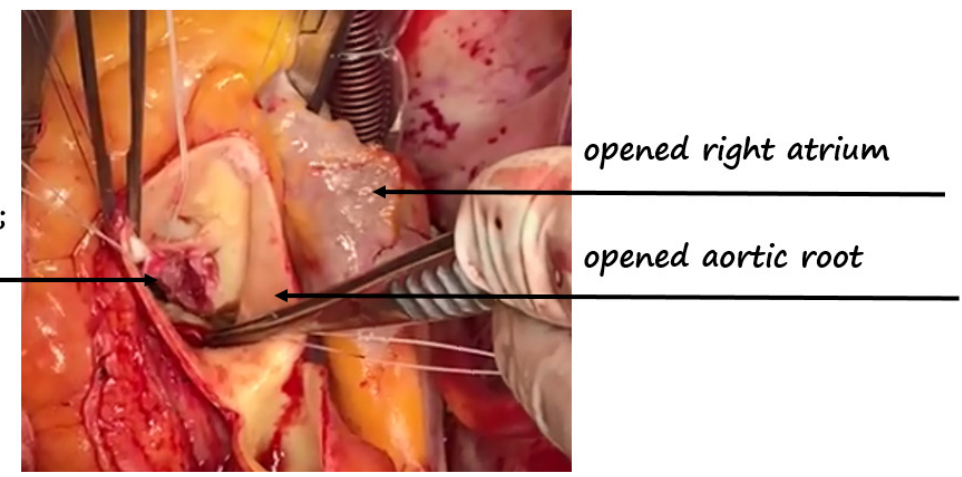

aortic abscess forced in the aortic wall by a Klemmer forceps, introduced through right atrium; a small fissuration in present

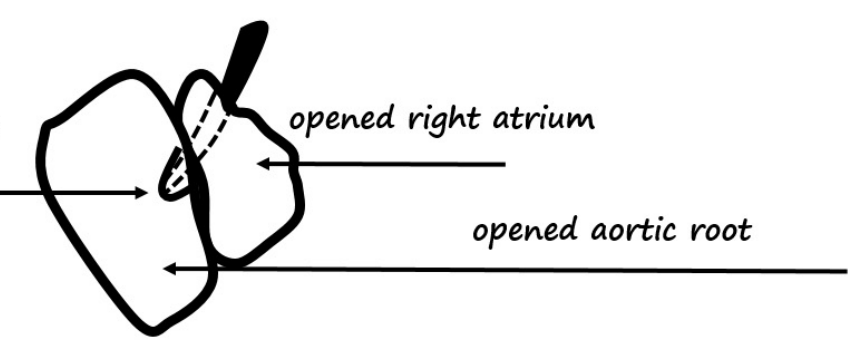

Figure 3. Top panel: surgical view with opened right atrium and aortic root. The Klemmer forceps were forced through the right atrium, showing the aortic abscess with a little fissure [3]. Bottom panel shows the relative pictogram.

Author Contributions: Conceptualization, G.T.; methodology, P.G., G.C. and F.B.; writing—original draft preparation, G.T., P.G., G.C. and F.B.; review and editing, E.P., G.E. and M.A.L.; supervision, M.A.L. All authors have read and agreed to the published version of the manuscript.

Funding: This research received no external funding.

Institutional Review Board Statement: This study was conducted in accordance with the Declaration of Helsinki and approved by Institutional Review Board of Samsung Medical Center (IRB file number 2021-04-127). 
Informed Consent Statement: This study was retrospective study based on electronic medical record review, and was exempted from consent through the Institutional Review Board.

Data Availability Statement: Data related to this study cannot be sent to the outside due to information security policies in the hospital.

Conflicts of Interest: The authors declare no conflict of interest.

\section{References}

1. Durack, D.T.; Lukes, A.S.; Bright, D.K. New criteria for diagnosis of infective endocarditis: Utilization of specific echocardiographic findings. Duke Endocarditis Serv. Am. J. Med. 1994, 96, 200-209. [CrossRef]

2. Otto, C.M.; Nishimura, R.A.; Bonow, R.O.; Carabello, B.A.; Erwin, J.P., 3rd; Gentile, F.; Jneid, H.; Krieger, E.V.; Mack, M.; McLeod, C.; et al. 2020 ACC/AHA Guideline for the Management of Patients With Valvular Heart Disease: Executive Summary: A Report of the American College of Cardiology/American Heart Association Joint Committee on Clinical Practice Guidelines. J. Am. Coll. Cardiol. 2021, 77, 450-500. [CrossRef] [PubMed]

3. Writing Committee; Pettersson, G.B.; Coselli, J.S.; Hussain, S.T.; Griffin, B.; Blackstone, E.H.; Gordon, S.M.; LeMaire, S.A.; Woc-Colburn, L.E. 2016 The American Association for Thoracic Surgery (AATS) consensus guidelines: Surgical treatment of infective endocarditis: Executive summary. J. Thorac. Cardiovasc. Surg. 2017, 153, 1241-1258. [CrossRef] [PubMed] 